\title{
Solid-Pseudopapillary tumor of the pancreas: Clinical and radiological features. Case report and review of the literature
}

\author{
Slim Jarboui ${ }^{1^{*}}$, Alifa Daghfous ${ }^{2}$, Karima Kacem $^{3}$, Lamia Rezgui Marhoul ${ }^{2}$ \\ ${ }^{1}$ Department of General Surgery, Sidi Bouzid Hospital, Sidi Bouzid, Tunisia; ${ }^{*}$ Corresponding Author: drslimjarboui@yahoo.fr \\ ${ }^{2}$ Department of Radiology, Trauma Center of Ben Arous, Ben Arous, Tunisia \\ ${ }^{3}$ Department of Hematology, Aziza Othmana Hospital, Tunis, Tunisia
}

Received 23 July 2013; revised 26 August 2013; accepted 10 September 2013

Copyright (C) 2013 Slim Jarboui et al. This is an open access article distributed under the Creative Commons Attribution License, which permits unrestricted use, distribution, and reproduction in any medium, provided the original work is properly cited.

\begin{abstract}
Solid-Pseudopapillary Tumor (SPT) of the pancreas is considered to be a rare pancreatic tumor that occurs in young females. Most SPTs are considered to be benign. However, the natural course of history has not yet been clarified. We reported a case of 25-year-old women with a patent history of splenectomy for hemolytic anemia, who presented intermittent abdominal pain and vomiting of 6 months ago. Physical examination revealed a hard mass of $8 \times 5 \mathrm{~cm}$ in the left upper quadrant of the abdomen. Routine laboratory tests were within the normal range. CTScan showed an $8 \times 6 \mathrm{~cm}$ lesion with irregular low density in the body and the tail of the pancreas. MRI showed a mass of $8 \mathrm{~cm}$, surrounded by a capsule, with the irregular intensity both in T1 and T2 enhanced-images. Distal pancreatictomy was done as a definitive treatment via a $\mathrm{Bi}$ sub costal incision. Histopathological examination confirmed the diagnosis of SPT. There was no vascular invasion or other malignant features. The resection margins are free of lesions. The post operative course was uneventful. During post operative follow-up of $\mathbf{2 4}$ months, there is no sign of recurrence on CT-scan. SPT is a rare condition with a low potential for malignancy and favorable prognosis. Surgical resection is generally curative. Characteristic CT and MRI scans combined with age and sex profile should be sufficient for the diagnosis and the decision to operate.
\end{abstract}

Keywords: Soli-Pseudopapillary Tumor; Distal Pancreatectomy; Pancreas

\section{INTRODUCTION}

Solid-Pseudopapillary Tumor (SPT) of the pancreas or Frantz tumor is a rare exocrine neoplastic tumor of the pancreas with a high prevalence in young women. It accounts for $0.13 \%-2.7 \%$ of all pancreatic tumors and it's generally considered as a tumor with a low malignant potential [1-4]. Although resection of the tumor provides a 5 -year survival rate of $97 \%$, local recurrence or distant metastases can occur in a significant number of patients [1,2,5-8].

For delineating the clinicopathological and radiological features, and the surgical therapy strategy of this tumor, we hereby report a clinical case with a review of the literature.

\section{CASE REPORT}

A 25-year-old woman with a past medical history of laparotomic splenectomy for hematologic anemia (mixed $\alpha$ and $\beta$ thalassemia) was transferred to our department for abdominal mass. She was complaining of intermittent epigastric pain with nausea and vomiting. An $8 \times 6 \mathrm{~cm}$ immobilized mass with distinct margin, and slight tenderness was palpable on the left upper quadrant. Neither jaundice nor enlarged superficial lymph nodes were seen.

Laboratory findings were normal except anemia with Hemoglobin at $8.5 \mathrm{~g} / \mathrm{dl}$ and increased level of ASAT (85 UI/l, normal < 45UI/L) and ASAT (103 UI/l, normal < 35 UI/l). Abdominal ultrasonography demonstrated a heterogeneous semi solid mass with cystic components adjacent to the left lobe of the liver. CT-scan delineated a well-demarcated solid-cystic lesion at the pancreatic tail surrounded by an integrated capsule with a diameter of $8 \times$ $6 \mathrm{~cm}$. The solid areas were in the central and the cystic areas were peripheral (Figure 1(a)). Intravenous contrast injection revealed slight enhancement of the cystic wall 
and solid part in arterial phase (Figure 1(b)), and moderate enhancement in portal phase (Figure 1(c)). No calcification was seen. MRI showed an $8 \mathrm{~cm}$ avoid solid-cystic mass of the pancreatic tail with distinct border. On T1 weight, heterogeneous iso intense lesion was

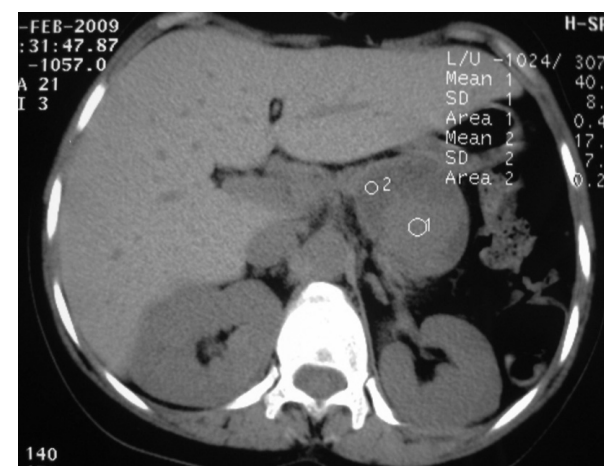

(a)

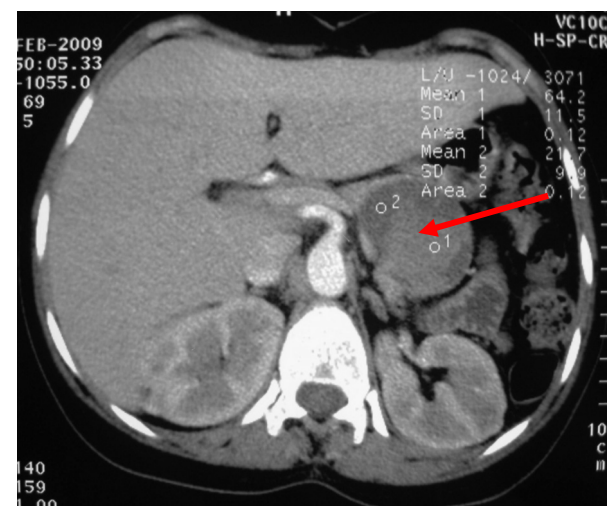

(b)

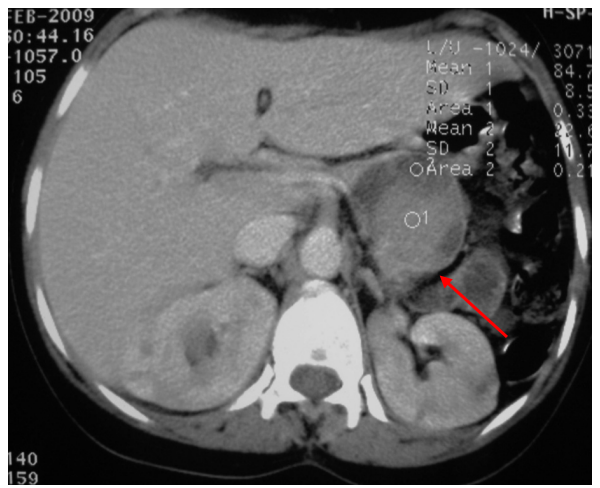

(c)

Figure 1. (a) Axial non enhanced CT-scan demonstrating a well-demarcated solid-cystic at the pancreatic tail surrounded by an integrated capsule with a diameter of $8 \times 6 \mathrm{~cm}$; (b) Arterial phase of contrast-enhanced CT demonstrating slight enhancement of the centrally solid portion of the lesion (Arrow); (c) Portal phase of contrast-enhanced CT demonstrating papillae-like enhancement of the solid portion and the peripheral cystic wall (Arrow). observed (Figure 2(a)), on T2 weight, papillae-like hypertensed and irregular hypointense area in the mass was seen (Figure 2(b)). The mass was irregularly enhanced with the significantly enhanced papillary solid centrally portion and the cystic wall after gadolinium injection (Figure 2(c)). There was no evidence of locoregional or distant spread on CT-scan or MRI.

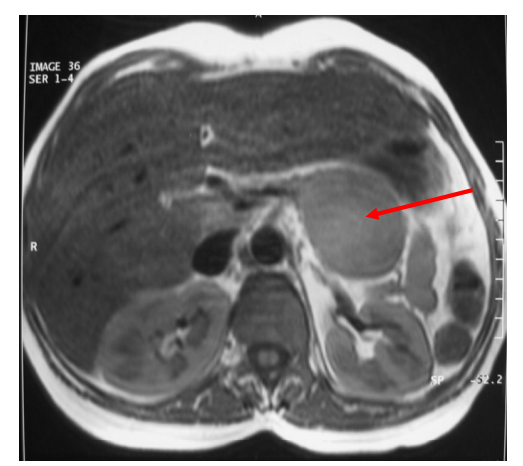

(a)

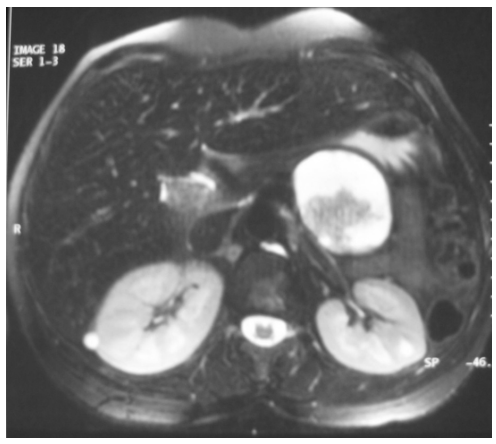

(b)

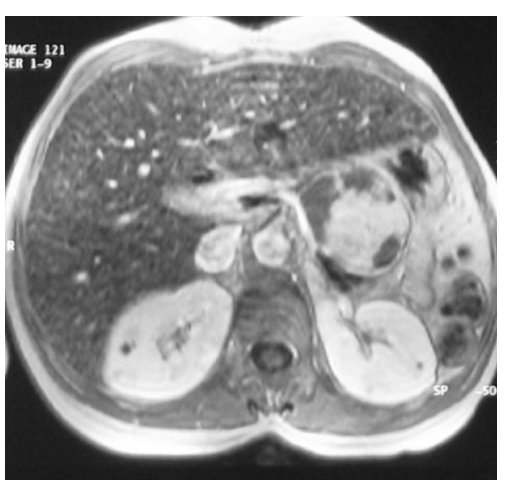

(c)

Figure 2. (a) T1 weigh showing isointense lesion in the pancreatic tail with hyper-intense hemorrhage in the centre (Arrow); (b) T2 Weigh MRI showing papillae-like hypo-intense and peripheral cystic hyper-intense areas in the mass; (c) Enhanced-MRI showing heterogeneous enhancement of the mass with obvious enhancement of the solid centrally portion and the wall of the cystic lesion. 
The patient was qualified to the scheduled surgical procedure. The Bi sub costal incision was performed. The tumor was resected completely via a distal pancreatectomy (Figure 3). The region of pancreatic tail was managed using the manual suture and the omental pack. The post operative period was uneventful. She was discharged 6 days after the surgical procedure.

On gross examination of the specimen, an $8 \times 6 \mathrm{~cm}$, oval, well defined and mixed solid-cystic mass was seen. Cut surface was grayish white, with areas of necrosis and hemorrhage. Surrounding pancreas at resection margins appeared normal. On histological examination, the tumor was very cellular and well-circumscribed without involvement of adjacent pancreas. The tumor cells were arranged in solid sheets and pseudopapillae. Mitotic figures were few. No vascular invasion was seen. Immunohistochemical staining of viementin, neuron-specific enolase (NSE) were positive, but negative reactions were found for chromogranine A, cytokeratine and CEA. With these histological features, final diagnosis of SPT of the pancreas was made.

24 months after surgery, the patient is asymptomatic and CT-scan shows no signs of recurrences or metastases.

\section{DISCUSSION}

SPTs of the pancreas are unusual neoplasms of the pancreas of uncertain histogenesis that occur mostly, but not exclusively in young women in the second or third decade. It is a rare neoplasm that has shown a progressive increase of incidence, passing from $0.17 \%-2.7 \%$ of 711 exocrine tumors of the pancreas in the 1980's, to $6 \%$ in recent report in 2003 [1-10]. Until 2006, there have been 718 cases in the English literature: female dominance has been found with a ratio of 10:1 and there was a mean age of 22 years [11]. In the review of 553 cases in Chinese literature, Peng-Fei Yu et al. had reported a mean age of patients of 27.2 years, and the male to female ratio was 1:8.37 [12].

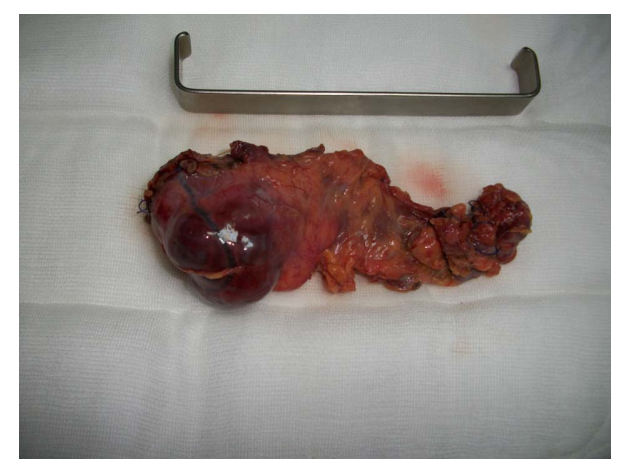

Figure 3. Operative specimen showing a wellcircumscribed mass occupying the tail of the pancreas.
The histogenesis of these epithelial neoplasms remains unclear. They have been suggested to have a ductal epithelial, neuroendocrine multipotent primordial cell, or even an extra pancreatic genital related cell origin [2,3, 7,12-17]. Deshpande et al. have described three cases of SPT of the ovary that resembling those of the pancreas [15]. The predominantly young female distribution of this tumor led to suggestions that estrogen and progesterone hormones may have a role in the pathogenesis $[1,7]$.

They are slow growing tumors with indolent course as was seen in our case. The diagnosis in most cases is based on compressive symptoms, pain or finding of a palpable mass, while about $20 \%$ to $30 \%$ of the patients, the diagnosis is made during abdominal imaging performed for others pathologies [1-3,5,7,12,13,18-22]. Even more unusual is, its presentation as an acute abdomen symptom with hemoperitoneum from spontaneous rupture or intratumoral bleeding [23-29]. They commonly located in the body and tail of pancreas also like our patient $[1-7,11,12,18]$. In the review of Peng-Fei Yu et al. $43.6 \%$ of tumors were localized in the body and tail of the pancreas [12].

SPT have not been associated with specific clinical laboratory test findings including serum tumor markers. The finding in the macroscopic pathological analysis corresponds to images in the CT-scans and MRI. Ultrasosnography shows the tumor as a well-defined mass consisting of solid as well cystic components $[1,8,14,18$, 25,28-31]. Typical SPT is characterized by a well-encapsulated mass with ranging amounts of intraluminal hemorrhage $[1,7,8,12,25]$. However, the tumor can have atypical appearance, such as metastasis, ductal obstruction, parenchymal and extra capsular invasion, simulation of islet cell tumor, intratumoral calcification, and occurrence in male patient. Knowledge of the spectrum of variable features observed in SPT is useful for differentiating this lesion from other pancreatic neoplasm and formulating the correct diagnosis. The classic CT features of SPT are a large well-encapsulated mass with varying solid and cystic components caused by hemorrhagic degeneration. Their mutual proportions can be various and the tumor can be differentiated from entirely solid to completely cystic [1,6,8,11,18,25-27,31,32]. Calcifications and enhancing solid areas may be present at the periphery of the mass. MRI typically shows a welldefined lesion with a mix of high and low signal intensity on T1 and T2 weighted images. T2-weighted images show a thick fibrous capsule, which is seen as a discontinuous rim of low signal intensity. Gadolinium enhanced dynamic MRI shows early peripheral heterogeneous enhancement of the solid portion with progressive fill-in [7,12,18,22,26,30-35]. Endoscopic ultrasound (EUS) may provide fine needle puncture biopsy with a supplemen- 
tary option of preoperative diagnosis. Fine-needle aspiration (FNA) can be effective in obtaining a preoperative diagnosis of SPT since the tumor has characteristic cytologic features [6,12,26,30,36-42]. On aspirated materials, the most frequent features are the presence of marked cellularity with pseudopapillary fragments composed of fibrovascular stalks lined with one to several layers of tumor cells [30,35,36,43-45]. In our case, clinical and radiological patterns are so specific to make preoperatively the diagnosis of SPT. Grossly, the tumors present as large, round, solitary masses that are usually well demarcated from the remaining pancreas. The cut surface of the tumors reveals lobulated, light brown solid areas admixed with zones of hemorrhage and necrosis as well as cystic spaces files with necrotic debris [1,3,5,8,12, $26,28,31,38]$. However, cystic changes can be less prominent in smaller tumors. Both a capsule and intratumoral hemorrhage are important clues to the diagnosis because these features are rarely found in other pancreatic neoplasms. Histologically, the tumors exhibit a solid monotonous pattern with sclerosis and a pseudopapillary pattern. Immunohistochemically, most tumor cells are positive for vimentin, alpfa-1-antitrypsin, alpha-1-antichymotrypsine, occasionally positive for neuron-specific enolase (NSE) and like in our patient, negative for cytokeratin, chromgranin, synaptophysin, and S-100 protein $[7,11,12,30,32]$. Recently, CD10 was demonstrated in SPT in a diffuse pattern, and therefore it can be helpful in differential diagnosis [11,12,20,30,45]. Many studies have used a large number of stains to document an immunohistochemical profile associated with SPT. They conclude that nuclear/cytoplasmic expression of Beta Catenin and loss of E-Cadherin can be also used in the diagnosis of SPT even in small biopsy specimens [11, 13,46-48]. The differential diagnosis includes pancreatic neuroendocrine tumor (PNET), acinar-cell carcinoma, papillary mucinous carcinomas, intraductal papillary mucinous tumor, pancreaticoblastoma and adenocarcinoma of the pancreas. PNET predominantly occur in older patients and can be associated with a variety of clinical syndromes. Additionally, these tumors express neuroendocrine markers such as chromgranin, NSE, and synaptophysin [6,7,18,20,21,30,32,35]. Acinar cell carcinoma occurring in a wide range of age is more commonly observed in males, and no predilection in location on the pancreas. Aspirates are cellular and composed only of acinar neoplastic cells containing enlarged nuclei with irregular membrane [7,21,30,32,35]. Papillary mucinous carcinoma is often presented as a single, large, unilocular cystic mass and the neoplastic cells are columnar with cytoplasmic vacuoles, prominent nucleoli and mucinous background. Also, the thick and viscid mucus almost always present in intraductal papillary mucinous tumor is an important feature that distinguishes this neoplasm from SPT [7,20,21,30]. Pancreaticoblastoma is a disease of childhood. Pancreatic adenocarcinoma is also seen in older patients. It does not grow as large as SPT. Calcification is quite unusual and cystic or hemorrhagic degeneration is uncommon [6,7,32].

Once the diagnosis of SPT is made, surgery is the first choice of treatment even in the case of distant hepatic metastases or local recurrence which is not contraindications for surgical therapy. The surgical treatment depended on the location of the neoplasm. Enucleation, partial pancreatectomy, distal pancreatectomy with or without splenectomy, Whipple procedure can be performed [1,2,5,7,8,10,12,23,25,27]. The surgical treatment has to be radical since the malignancy can only be defined by postoperative histological examination. Overall 5-year survival is high as $97 \%$ in patients undergoing surgical resection [1,4-7,12,21,25-28]. Metastases develop in less than $15 \%$ of cases and the liver is the most common site of metastasis [8,10,20,22,23,27,29,34,35, 45,49-55]. Liver metastatic lesions can be multiple but are generally solitary and may be amenable to resection. Given the reports that even patients with SPT with local recurrence as well as liver and peritoneal metastasis, could still have long-term survival, the presence of metastases is not a contraindication for surgery $[1,19,23$, $35,48,49]$. Several features such as old age, male patient, invasion into a capsule or adjacent normal pancreatic parenchyma, and both vascular and perineural invasion, are described as associated with an increase in potential for malignancy [7,8,10,22,27,28,35,50-55]. Yang et al. [54] in their study of 26 consecutive SPT concluded that there were no significant association between clinicopathological features and malignancy. High proliferative index (KI67) may predict outcome of malignant SPT. Tang et al. [8] in their review of 36 consecutive patients, they described two cases of SPT exhibiting an aggressive clinical course and both died of disease at 6 and 1 months after diagnosis. The pathologic features of these patients included a diffuse growth pattern, extensive tumor necrosis, significant nuclear atypia, high mitosis rate and in one case a component of sarcomatoid carcinoma. Nishihara et al. [9] have reported that venous invasion, diffuse growth pattern, extensive tumor necrosis, significant nuclear atypia and high mitotic rate are indication of aggressive behavior. Mackado et al. [36] in their series of 34 patients demonstrated that SPT in male patients have distinct pattern of onset and aggressiveness when compared with female patients. Geers et al. [16] suggest that Galactin 3 is a useful marker to distinguish SPT from PNET, and it is also indicator of behavior because of its low expression is associates with metastatic spreading. Our patient have nor of these predictive signs of malignancy in histological examination. In fact, the follow up of a large number of cases has shown that the majority of 
SPTs are benign. They have been differentiated by the WHO classification into SP neoplasms with borderline malignant potential and SP carcinomas [46]. Criteria for this distinction are angioinvasion, perineural invasion and deep invasion in the surrounding pancreatic parenchyma. There is limited experience regarding chemotherapy and radiotherapy with or without the presence of metastatic disease [2,11]. Regular follow-up is mandatory for all patients having undergone potentially curative resection and particularly on patients with tumor with histology suggesting a possible malignant behavior.

\section{CONCLUSION}

In conclusion, SPT is a low grade tumor with a good prognosis. The presence of a huge mass in the pancreas of young female should prompt suspicion for a SPT. Given its low malignant potential, and the presence of specific radiographic patterns, its diagnosis should be accurate, as the radical surgical treatment is effective. Surgical resection is the mainstay of treatment and is possible in the majority of cases.

\section{REFERENCES}

[1] Chen, S.-Q., Zou, S.-S., Dai, Q.-B., et al. (2008) Clinical analysis of solid-pseudopap illary tumor of the pancreas: Report of 15 cases. Hepatobiliary \& Pancreatic Diseases International, 7, 196-200.

[2] Arkuszewski, P., Pasieka, Z., Srebrzynski, A., et al. (2011) Solid pseudopapillary tumor of the pancreas in a young woman-case report. Polski Przeglad Chirurgiczny, 83, 389-391. http://dx.doi.org/10.2478/v10035-011-0062-0

[3] Kloppel, G., Morohoshi, T., John, H.D., et al. (1981) Solid and cystic acinar cell tumor of the pancreas. A tumor in young women with favorable prognosis. Virchows Archiv. A, Pathological Anatomy and Histology, 392, 171-183. http://dx.doi.org/10.1007/BF00430819

[4] Kloppel, G., Solcia, E., Longnecker, D.S., et al. (1996) Histological typing of tumors of the exocrine pancreas. In: World Health Organisation, Ed., International Histological Classification of Tumors, 2nd Edition, Springer, Berlin Heidelberg New York, 120-128.

[5] Lam, K.Y., Lo, C.Y. and Fan, S.T. (1999) Pancreatic solidcystic-pseudopapillary tumor: Clinicopathologic features in eight patients from Hong Kong and review of the literature. World Journal of Surgery, 10, 1045-1050. http://dx.doi.org/10.1007/s002689900621

[6] Nakahara, K., Kobayashi, G., Fujita, N., et al. (2008) Solid-pseudopapillary tumor of the pancreas showing a remarkable reduction in size over the 10-year follow-up. Internal Medicine, 47, 1335-1339.

http://dx.doi.org/10.2169/internalmedicine.47.0767

[7] Shaikh, S., Arya, S., Ramadwar, M., et al. (2008) Three Cases of unusual solid-pseudopapillary tumors. Can radiology and histology aid decision-making. JOP, 9, 150159.
[8] Tang, L.H., Aydin, H., Brennan, M.F., et al. (2005) Clinically aggressive solid-pseudopapillary tumors of the pancreas: A report of two cases with components of undifferentiated carcinoma and a comparative clinicopathologic analysis of 34 conventional cases. The American Journal of Surgical Pathology, 29, 512-519. http://dx.doi.org/10.1097/01.pas.0000155159.28530.88

[9] Nishihara, K., Nagoshi, M., Tsuneyoshi, M., et al. (1993) Papillary cystic tumors of the pancreas, assessment of their malignant potential. Cancer, 71, 82-92. http://dx.doi.org/10.1002/1097-0142(19930101)71:1<82:: AID-CNCR2820710114>3.0.CO;2-Y

[10] Kang, C.M., Kim, K.S., Choi, J.S., et al. (2006) Solidpseudopapillary tumor of the pancreas suggesting malignnant potential. Pancreas, 3, 276-280. http://dx.doi.org/10.1097/01.mpa.0000202956.41106.8a

[11] Mulkeen Abby, L., Yoo Peter, S. and Cha, C.H. (2006) Less common neoplasm of the pancreas. World Journal of Gastroenterology, 12, 3180-3185.

[12] Yu, P.F., Hu, Z.H., Wang, X.B., et al. (2010) Solid pseudopapillary tumor of the pancreas: A review of 553 cases in Chinese literature. World Journal of Gastroenterology, 16, 1209-1214. http://dx.doi.org/10.3748/wjg.v16.i10.1209

[13] Khan, F.Y., El-Hiday, A. and Morad, N.A. (2007) Plummer-vinson syndrome associated with solid-pseudopapillary tumor of the pancreas. Chinese Medical Journal, 120, 1553-1555.

[14] Cheuk, W., Beavon, I., Chui, D.T., et al. (2011) Extrapancreatic solid pseudopapillary neoplasm: Report of a case of primary ovrian origin and review of the literature. International Journal of Gynecologic Pathology, 30, 539543. http://dx.doi.org/10.1097/PGP.0b013e31821724fb

[15] Deshpande, V., Oliva, E. and Young, R.H. (2010) Solid pseudopapillary neoplasm of the ovary: A report of 3 primary ovarian tumors resembling those of the pancreas. The American Journal of Surgical Pathology, 34, 15141520. http://dx.doi.org/10.1097/PAS.0b013e3181f133e9

[16] Geers, C., Moulin, P., Gigot, J.F., et al. (2006) Solid and pseudopapillary tumor of the pancreas-Review and new insights into pathogenesis. The American Journal of Surgical Pathology, 30, 1243-1249.

http://dx.doi.org/10.1097/01.pas.0000213311.28682.b2

[17] Mao, C., Guvendi, M., Domenico, D.R., et al. (1995) Papillary cystic and solid tumors of the pancreas: A pancreatic embryonic tumor? Studies of three cases and cumulative review of the world's literature. Surgery, 118, 821-828. http://dx.doi.org/10.1016/S0039-6060(05)80271-5

[18] Choi, J.Y., Kim, M.J., Kim, J.H., et al. (2006) Solid pseudopapillary tumor of the pancreas: Typical and atypical manifestations. AJR, 187, 178-186. http://dx.doi.org/10.2214/AJR.05.0569

[19] Raffel, A., Cupisti, K., Krausch, M., et al. (2004) Therapeutic strategy of papillary cystic and sold neoplasm (PCSN): A rare non-endocrine tumor of the pancreas in children. Surgical Oncology, 13, 1-6.

http://dx.doi.org/10.1016/j.suronc.2003.09.003

[20] Bostanoglu, S., Otan, E., Akturan, S., et al. (2009) Frantz's 
tumor (solid-pseudopapillary tumor) of the pancreas. A case report. JOP, 10, 209-211.

[21] Kamat, R.N., Naik, L.D., Joshi, R.M., et al. (2008) Solid pseudopapillary tumor of the pancreas. Indian Journal of Pathology and Microbiology, 51, 271-273. http://dx.doi.org/10.4103/0377-4929.41692

[22] Sperti, C., Berselli, M., Pasquali, C., et al. (2008) Aggressive behaviour of solid-pseudopapillary tumor of the pancreas in adults: A case report and review of the literature. World Journal of Gastroenterology, 14, 960-965. http://dx.doi.org/10.3748/wjg.14.960

[23] Vergauwen, W., Op de Beeck, B., Hagendorens, M., et al. (2010) A solid pseudopapillary tumor of the pancreas presenting after an abdominal trauma. Acta Chirurgica Belgica, 110, 390-393.

[24] Hansson, B., Hubens, G., Hagendorens, M., et al. (1999) Frantz's tumor of the pancreas presenting as a post-traumatic pancreatic pseudocyst. Acta Chirurgica Belgica, 99, 82-84.

[25] Frago, R., Fabregat, J., Jorba, R., et al. (2006) Solid pseudopapillary tumors of the pancreas: Diagnosis and curative treatment. Revista Espanola de Enfermedades Digestivas, 98, 809-816. http://dx.doi.org/10.4321/S1130-01082006001100002

[26] Zuriarrain, A., Nir, I., Bocklage, T., et al. (2011) Pseudopapillary tumor of the pancreas in a 17-year-old girl. Journal of Clinical Oncology, 29, 395-396. http://dx.doi.org/10.1200/JCO.2010.33.5364

[27] Chang, H., Gong, Y.I., Xu, J., et al. (2010) Clinical strategy for the management of solid pseudopapillary tumor of the pancreas. Aggressive or less? International Journal of Medical Sciences, 7, 309-613. http://dx.doi.org/10.7150/ijms.7.309

[28] Yong, F., Fu, D.L., Jin, C., et al. (2008) Clinical experiences of solid pseudopapillary tumors of the pancreas in China. Journal of Gastroenterology and Hepatology, 23, 1847-1851. http://dx.doi.org/10.1111/j.1440-1746.2008.05526.x

[29] Salvia, R., Bassi, C., Festa, L., et al. (2007) Clinical and biological behavior of pancreatic solid-pseudopapillary tumors: Report on 31 consecutive patients. Journal of Surgical Oncology, 95, 304-310. http://dx.doi.org/10.1002/jso.20685

[30] Goleman, K.M., Doherty, M.C. and Bigler, S.A. (2003) Solid-pseudopapillary tumor of the pancreas. Radiographics, 23, 1644-1648. http://dx.doi.org/10.1148/rg.236035006

[31] Patil, T.B., Shrikhande, S.V., Kanhere, H.A., et al. (2006) Solid pseudopapillary neoplasm of the pancreas: A single institution experience of 14 cases. $H P B, \mathbf{8}, 148-150$. http://dx.doi.org/10.1080/13651820510035721

[32] Yu, C.C., Tseng, J.H., Yeh, C.N., et al. (2007) Clinicopathological study of solid and pseudopapillary tumor of the pancreas: Emphasis on magnetic resonance imaging findings. World Journal of Gastroenterology, 13, 18111815.

[33] Dong, D.J. and Zhang, S.Z. (2006) Solid-pseudopapillary tumor of the pancreas: CT and MRI features of 3 cases.
Hepatobiliary \& Pancreatic Diseases International, 5 , 300-304.

[34] Lee, J.H., Yu, J.S., Kim, H., et al. (2008) Solid pseudopapillary carcinoma of the pancreas: Differentiation from benign solid pseudopapillary tumour using CT and MRI. Clinical Radiology, 63, 1006-1014.

http://dx.doi.org/10.1016/j.crad.2008.04.007

[35] Huang, H.L., Shih, S.C., Chang, W.H., Chen, M.J., Chan, Y.J., et al. (2005) Solid-pseudopapillary tumor of the pancreas: Clinical experience and literature review. World Journal of Gastroenterology, 11, 1403-1409.

[36] Machado, M.C., Machado, M.A., Bacchella, T., et al. (2008) Solid pseudopapillary neoplasm of the pancreas: Distinct patterns of onset, diagnosis, and prognosis for male versus female patients. Surgery, 143, 29-34. http://dx.doi.org/10.1016/j.surg.2007.07.030

[37] Kosuke, M., Masahiko, H., Shinya, A., et al. (2010) Small solid pseudopapillary tumor of the pancreas in a 32-year-old man: Report of a case. Surgery Today, 40, 772-776. http://dx.doi.org/10.1007/s00595-009-4139-x

[38] Santini, D., Poli, F. and Lega, S. (2006) Solid-papillary tumors of the pancreas: Histopathology. Journal of the Pancreas, 7, 131-136.

[39] Crawford Jr., B.E. (1998) Solid and papillary epithelial neoplasm of the pancreas, diagnosis by cytology. Southern Medical Journal, 91, 973-977. http://dx.doi.org/10.1097/00007611-199810000-00017

[40] Trivedi, N., Sharma, U., Das, P.M., et al. (1999) FNAC of papillary and solid epithelial neoplasm of pancreas-A case report. Indian Journal of Pathology \& Microbiology, 42, 369-372.

[41] Naresh, K.N., Borges, A.M., Chinoy, R.F., et al. (1995) Solid and papillary epithelial neoplasm of the pancreas. Diagnosis by fine needle aspiration cytology in four cases. Acta Cytologica, 39, 489-493.

[42] Burford, H., Baloch, Z., Liu, X., Jhala, D., et al. (2009) A limited immunohistochemical panel to distinguish pancreatic endocrine neoplasm from solid pseudopapillary neoplasm of the pancreas on endoscopic ultrasoundguided fine-needle aspirates of the pancreas. American Journal of Clinical Pathology, 132, 831-839. http://dx.doi.org/10.1309/AJCPVT8FCLFDTZWI

[43] Pezzi, C.M., Schuerch, C., Erlandson, R.A., et al. (1988) Papillary-cystic neoplasm of the pancreas. Journal of Surgical Oncology, 37, 278-285. http://dx.doi.org/10.1002/jso.2930370414

[44] Salla, C., Chatzipantelis, P., konstantinou, P., et al. (2007) Endoscopic ultrasound-guides fine-needle aspiration cytology diagnosis of solid pseudopapillary tumor of the pancreas: A case report and literature review. World Journal of Gastroenterology, 13, 5158-5163.

[45] Klimstra, D.S., Wenig, B.M. and Heffess, C.S. (2000) Solid-pseudopapilary tumor of the pancreas: A typically cystic carcinoma of low malignant potential. Seminars in Diagnostic Pathology, 17, 66-80.

[46] Jai, H.G. (2008). A spindle cell predominant pancreatic solid-pseudopapillary tumor. Yonsei Medical Journal, 49, 672-675. http://dx.doi.org/10.3349/ymj.2008.49.4.672 
[47] Kim, M.J., Jang, S.J. and Yu, E. (2008) Loss of E-cadherin and cytoplasmic-nuclear expression of beta-catenin are the most useful immunoprofiles in the diagnosis of solid-pseudopapillary neoplasm of the pancreas. Human Pathology, 39, 251-258. http://dx.doi.org/10.1016/j.humpath.2007.06.014

[48] Tiemann, K., Kosmahl, M., Ohlendorf, J., et al. (2006) Solid pseudopapillary neoplasms of the pancreas are associated with FLI-1 expression, but not with EWS/FLI-1 translocation. Modern Pathology, 19, 1409-1413.

[49] Tanaka, Y., Kato, K., Notohara, K., et al. (2001) Frequent $\beta$-catenin Mutation and cytoplasmic/nuclear accumulation in pancreatic solid-pseudopapillary neoplasm. Cancer Research, 61, 8401-8404.

[50] Tipton, S.G., Smyrk, T.C., Sarr, M.G., et al. (2006) Malignant potential of solid pseudopapillary neoplasm of the pancreas. British Journal of Surgery, 93, 733-737. http://dx.doi.org/10.1002/bjs.5334

[51] Yoon, D.Y., Hines, O.J., Bilchik, A.J., et al. (2001) Solid and papillary epithelial neoplasm of the pancreas: Ag- gressive resection for cure. The American Journal of Surgery, 67, 1195-1199.

[52] Nakagohri, T., Kinoshita, T., Konishi, M., et al. (2008) Surgical outcome of solid pseudopapillary tumor of the pancreas. Journal of Hepato-Biliary-Pancreatic Surgery, 15, 318-321.

http://dx.doi.org/10.1007/s00534-007-1264-z

[53] Siquini, W., Marmorale, C., Guercioni, G., et al. (2006) Solid pseudopapillary tumor of the pancreas. A report of 3 cases and e review of the literature. Chirurgia Italiana, 58, 235-245.

[54] Yang, F., Jin, C., Long, J., et al. (2009) Solid pseudopapillary tumor of the pancreas: A case series of 26 consecutive patients. The American Journal of Surgery, 198, 210-215. http://dx.doi.org/10.1016/j.amjsurg.2008.07.062

[55] Nagri, S., Abdu, A., Ananad, S., et al. (2007) Liver metastasis four years after Whipple's resection for solidpseudopapillary tumor of the pancreas. Journal of Pancreas, 8, 223-227. 